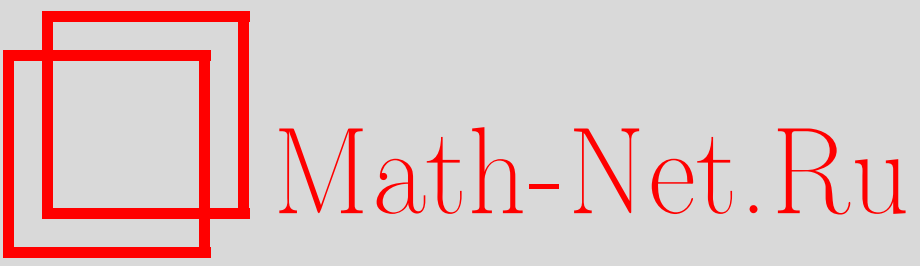

В. Н. Силаев, О ленточной размерности кольца формальных степенных рядов, УМН, 2001, том 56, выпуск 4, 157-158

DOI: https://doi.org/10.4213/rm431

Использование Общероссийского математического портала Math-Net.Ru подразумевает, что вы прочитали и согласны с пользовательским соглашением

http://www.mathnet.ru/rus/agreement

Параметры загрузки:

IP: 35.173 .219 .12

26 апреля 2023 г., $14: 21: 13$ 


\title{
О ЛЕНТОЧНОЙ РАЗМЕРНОСТИ КОЛЬЦА ФОРМАЛЬНЫХ СТЕПЕННЫХ РЯДОВ
}

\author{
В.Н. СИЛАЕВ
}

В 1991 г. Гудерл, Менал и Монкази доказали [1], что любая алгебра $A$ с 1 над полем $F$ может быть вложена в алгебру $B(F)$ всех $(\omega \times \omega)$-матриц над полем $F$, в каждой строке и каждом столбце которых содержится лишь конечное число отличных от нуля элементов. В [2] О'Мира и Ханнах ввели понятие кривой роста, определенное для любой матрицы из $B(F)$. Использовав результат Гудерла, Менала и Монкази, описанный выше, они получили функцию ленточной размерности. Там же ими был сформулирован список открытых проблем по теории ленточной размерности. Одним из подходов к решению некоторых из них было изучение возможности вложения кольца формалшных степенных рядов $F[[x]]$ в кольцо матриц $O(1)$-роста, что и исследуется в данной работе. Напомним основные определения.

Для любого элемента $x$ из алгебры $B(F)$ функция $g(n)$, определенная на множестве натуральных чисел, называется кривой роста матрицы $x$, если для каждого значения $n \in \mathbb{N} x(i, n)=$ $x(n, i)=0$ для всех номеров $i$ таких, что $i>n+g(n)$. Говорят, что матрица $x \in B(F)$ имеет noрядок роста $g(n)$ (или что $x$ имеет $O(g(n))$-рост), где $g: \mathbb{N} \rightarrow \mathbb{R}^{+}$, если существует постоянная $c>0$ такая, что функция $c g(n)$ является кривой роста для матрицы $x$.

Если $A$-подалгебра в $B(F)$ и каждый элемент из $A$ имеет $O(\Phi(n))$-рост, то говорят, что сама алгебра $A$ имеет $O(\Phi(n))$-рост. Матрицы с кривой роста вида $f(n)=c n^{\alpha}$, где $\alpha$-произвольное фиксированное значение, $\alpha \in[0,1]$, образуют подалгебру в $B(F)$, обозначаемую $G(\alpha)$. Введем также обозначение $W_{\alpha}(c)=\left\{x \in B(F) \mid x\right.$ имеет кривую роста $\left.c n^{\alpha}\right\}, W_{\alpha}(c)$ является подпространством $B(F)$.

ОПРеДЕЛЕНИЕ. Ленточной размерностью (bw. $\operatorname{dim})$ алгебры $A$ называется величина $\inf \{\alpha \in \mathbb{R}, \alpha \geqslant 0 \mid A$ вкладьвается в $G(\alpha)\}$.

Функция ленточной размерности принимает значения в промежутке $[0,1]$, причем все значения из $[0,1]$, на множестве алгебр с 1 над полем $F$, как показано в [2].

Теорема 1. Для любого поля $F \quad$ bw. $\operatorname{dim} F[[x]]=0$.

ДокАЗАТЕльство. Нетрудно показать, что $F[[x]] \subset G(r)$ для любого $r>0$. Тогда так как $r \in[0,1]$, то $0 \leqslant \inf \{r \in \mathbb{R}, r \geqslant 0 \mid F[[x]]$ вкладывается в $G(r)\} \leqslant 0$, откуда bw. $\operatorname{dim} F[[x]]=0$, что и требуется доказать.

Лемма 1. Пусть $|F|=L<\infty, A \in B(F) ;\left\{A, A^{2}, \ldots, A^{n}, \ldots\right\} \subset W_{k}(0)$. Тогда найдется многочлен $P(x) \neq 0, \operatorname{deg} P(x) \leqslant L(2 k+2)(2 k+1)$ такой, что $P(A)=0$.

ДоказАтельство. Рассмотрим $A$ как оператор на счетномерном пространстве $V$ с фиксированным базисом $\left\{e_{i}\right\}_{i=1}^{\infty}$. Тогда для любого $i$ справедливо $\left\{e_{i}, A e_{i}, A^{2} e_{i}, \ldots\right\} \subset\left\langle e_{i-k}, \ldots\right.$, $\left.e_{i}, \ldots, e_{i+k}\right\rangle$. Отсюда следует, что найдется многочлен $P_{i}(x) \neq 0, \operatorname{deg} P_{i}(x) \leqslant 2 k+1$, такой, что $P_{i}(A) e_{i}=0$. Количество различных ненулевых многочленов степени не больше $2 k+1$ над полем, состоящим из $L$ элементов, не превосходит $(2 k+2) L$. Взяв их произведение, получим многочлен, обладающий искомыми свойствами, степени не болшше $(2 k+2)(2 k+1) L$. Лемма доказана.

Teоpema 2. $F[[x]] \not \subset G(0)=\{x \in B(F) \mid x$ имеет кривую роста $C=C(x)$ - константа, зависящая от $x\}$. Другими словами, не существует вложения $F[[x]]$ в кольцо DFM счетномерных матрии с конечным числом ненулевых над- и поддиагоналей.

ДокАЗАтЕльство. А) Предположим, что существует такое вложение $\varphi: F[[x]] \rightarrow G(0)$. Обозначим через $A$ матрицу $\varphi(x)$. Матрицы из $G(0)$ можно рассматривать как операторы в счетномерном пространстве $V$, если там фиксировать базис. Теперь для любого $v \neq 0$ рассмотрим ряд векторов $v, A v, A^{2} v, A^{3} v, \ldots$. Для него есть две возможности: а) $v, A v, A^{2} v, A^{3} v, \ldots, A^{n} v, \ldots$ - линейно независимая система векторов; б) $v, A v, A^{2} v, A^{3} v, \ldots, A^{n} v, \ldots$ - линейно зависимая система векторов. Тогда существует многочлен $P(x) \neq 0$ такой, что $P(A) v=0$. Выберем многочлен наименьшей степени с единичным старшим коэффициентом. Он существует и единственен для 
каждого $v$; обозначим его $P_{v}(x)$. Далее, $P_{v}(x)=x^{n}\left(a_{0}+\cdots+a_{k-1} x^{k-1}+x^{k}\right)=x^{n} P_{v}^{\prime}(x)$, где $a_{0} \neq 0, P_{v}^{\prime}(x)$ - обратим в $F[[x]]$. Тогда имеем $\varphi\left(P_{v}^{\prime-1}(x)\right) \varphi\left(P_{v}^{\prime}(x) x^{n}\right) v=\varphi\left(x^{n}\right) v$ $=A^{n} v=0$. Отсюда так как степень многочлена выбирали наименьшей, то $P_{v}(x)=x^{n}$, $A^{n} v=0,\left\{v, A v, \ldots, A^{n-1} v\right\}$ - линейно независимая система. Таким образом, для любого $v \neq 0$ возможны два случая: а) $\{v, A v, \ldots\}$ - линейно независимая система; б) $A^{n} v=0$, $\left\{v, \ldots, A^{n-1} v\right\}$ - линейно независимая система.

Б) Введем обозначение $V_{0}=\left\{v \in V \mid \exists n \in \mathbb{N} A^{n} v=0\right\}$. Тогда $V_{0}$ - подпространство $V$, $A V_{0} \subset V_{0}$.

Лемма 2. Для любого $f(x)=a_{0}+a_{1} x+\cdots+a_{n} x^{n}+\cdots \in F[[x]]$ оператор $f(A)=a_{0}+$ $a_{1} A+\cdots+a_{n} A^{n}+\cdots$ определен корректно на $V_{0} u \varphi(f)=f(A)$ на $V_{0}$.

ДокаЗАТЕЛЬСТво. Для любого $v \in V_{0} f(A) v$ определено корректно и $\left.\varphi(f) v=f(A)\right) v$. Таким образом, получаем, что $\varphi(F[[x]]) V_{0} \subset V_{0}$.

В) Поэтому можно рассмотреть действие $\varphi(F[[x]]): V / V_{0}$. Допустим, что $V / V_{0} \neq 0$. Возьмем произвольный $v, 0 \neq v \in V / V_{0}$. Покажем, что тогда для любого $f(x) \in F[[x]], f(x) \neq 0$, вьполняется $\varphi(f) v \neq 0$. Действительно, $f(x)=x^{m}\left(a_{m}+a_{m+1} x+\cdots\right)$, где $a_{m} \neq 0 ; \varphi(f) v=$ $\varphi\left(\left(a_{m}+a_{m+1} x+\cdots\right)\right) \varphi\left(x^{m}\right) v \neq 0$, так как $A=\varphi(x)$ - инъективен на $V / V_{0}$ и ненулевые векторы переводит в ненулевые, а оператор $\varphi\left(\left(a_{m}+a_{m+1} x+\cdots\right)\right)$ обратим на $V / V_{0}$, поскольку ряд $\left(a_{m}+a_{m+1} x+\cdots\right)$ обратим в $F[[x]]$. Выберем континуальньй базис $\left\{f_{\nu}\right\}$ в $F[[x]]$. Тогда $\left\{\varphi\left(f_{\nu}\right) v\right\}$ линейно независимы в силу $\varphi(f) v \neq 0$. Таким образом, получаем противоречие со счетномерностью $V / V_{0}$. Отсюда следует, что $V / V_{0}=0$ и $V=V_{0}$.

Г) Лемма 3. Пусть $A=\varphi(x), \operatorname{mozдa~}\left\{A, A^{2}, \ldots, A^{n}, \ldots\right\} \not \subset W_{k}(0)$ ни для какого фиксированного $k$.

ДокаЗАТЕльство. Выберем в $V=V_{0}$ базис $\left\{e_{i}\right\}_{i=1}^{\infty}$. Тогда для любого $i$ выполняется $\left\{e_{i}, A e_{i}, A^{2} e_{i}, \ldots\right\} \subset L$, где $L=\left\langle e_{i-k}, \ldots, e_{i}, \ldots, e_{i+k}\right\rangle$. Отсюда следует, что найдется многочлен $P_{i}(x) \neq 0, \operatorname{deg} P_{i}(x) \leqslant 2 k+1$, такой, что $P_{i}(A) e_{i}=0$. Мы знаем, что минимальный аннулирующий $A$ на $e_{i}$ многочлен имеет вид $x^{n_{i}}$, откуда $A^{n_{i}} e_{i}=0$ и для любого $i n_{i} \leqslant 2 k+1$. Следовательно, $A^{2 k+1} e_{i}=0$ для любого $i$. Отсюда $A^{2 k+1}=0$-противоречие с тем, что $\varphi-$ вложение.

Д) Таким образом, (i) ненулевые матричные элементы матриц $A^{n}$ удаляются от главной диагонали с ростом $n$; (ii) в то же время для любого $N \in \mathbb{N}$ найдется $M \in \mathbb{N}$ такое, что $A^{M^{\prime}} e_{i}=0$ для любого $i, 1 \leqslant i \leqslant N$, и для любого $M^{\prime}, M^{\prime} \geqslant M$ (поскольку $V=V_{0}$ ). Выберем, пользуясь (i) и (ii), последовательности натуральных чисел $\left\{n_{l}\right\}_{l=1}^{\infty},\left\{k_{l}\right\}_{l=1}^{\infty},\left\{i_{l}\right\}_{l=1}^{\infty},\left\{j_{l}\right\}_{l=1}^{\infty}$ такие, что для любого $l: n_{l+1}>n_{l}, k_{l+1}>k_{l}$, и выполнены следуюшие свойства: $\left(A^{n_{l}}\right)_{i_{l} j_{l}} \neq 0,\left|i_{l}-j_{l}\right|=k_{l}$; $A^{n} \in W_{k_{l}-1}(0)$ для любого $n, n<n_{l}$; для любого $m, m<l,\left(A^{n_{l}}\right)_{i_{m} j_{m}}=0$. Тогда корректно определенная по п. Б) матрица $\left(A^{n_{1}}+A^{n_{2}}+\cdots+A^{n_{l}}+\cdots\right)$ будет обладать следующими свойствами: для любого $l$ справедливо $\left(A^{n_{1}}+A^{n_{2}}+\cdots+A^{n_{l}}+\cdots\right)_{i_{l} j_{l}}=\left(A^{n_{l}}\right)_{i_{l} j_{l}} \neq 0$; для $f(x)=x^{n_{1}}+x^{n_{2}}+\cdots+x^{n_{l}}+\cdots \in F[[x]]$ вьполнено $\varphi(f)=A^{n_{1}}+A^{n_{2}}+\cdots+A^{n_{l}}+\cdots$. Получаем, что $(\varphi(f))_{i_{l} j_{l}} \neq 0$, причем $\left|i_{l}-j_{l}\right| \rightarrow \infty$. Отсюда следует, что $(\varphi(f)) \notin G(0)$, что является противоречием с исходным предположением. Теорема 2 доказана.

\section{СПИСОК ЛИТЕРАТУРЫ}

[1] K. R. Goodearl, P. Menal, J. Moncasi // J. Algebra. 1993. V. 156. № 2. P. 407-432. [2] J. Hannah, K. C. O'Meara. A new measure of growth for countable-dimensional algebras I // Trans. Amer. Math. Soc. 1995. V. 347. № 1. P. 117-136.

Московский государственный университет им. М. В. Ломоносова
Принято редколлегией 01.06 .2001 